\title{
Effets d'un apport de méthionine et de lysine dans l'intestin sur la croissance, la rétention azotée et les paramètres plasmatiques de jeunes bovins
}

\author{
Y Geay, P Valma
}

\author{
avec la collaboration de Rd et Rt Jailler \\ INRA Clermont-Ferrand-Theix, laboratoire croissance et métabolisme des herbivores, \\ 63122 Saint-Genès-Champanelle, France
}

\begin{abstract}
Summary - Effect of post-ruminal infusion of methionine and lysine on growth, nitrogen retention and plasma parameters in young bulls. The same amount of nitrogen $12.7 \%$ of $\mathrm{N}$ intake) was administered by post-ruminal infusion to young bulls $(13 \mathrm{~g}$ methionine $-13 \mathrm{~g}$ lysine vs $\mathbf{4 4} \mathrm{g}$ sodium glutamate). Supplementing animals with limiting amino acids (methionine and lysine) improved the growth rate and the $N$ balance. This experiment shows that the specific requirements of young bulls for methionine and lysine should be taken into account when establishing diet composition and $\mathrm{N}$ allowances.
\end{abstract}

Les besoins en acides aminés indispensables (AAI) des bovins en croissance sont généralement couverts par l'apport microbien (Owens, 1986). Mais la synthèse d'azote aminé microbien a des limites $(18,6 \mathrm{~g}$ d'N/kg MOF; Vérité et al, 1987). Elle pourrait donc, dans certains cas, être inférieure aux besoins de l'animal lorsque celui-ci a une rétention protéique élevée, et ne pas être suffisamment complétée au niveau intestinal par les AAl d'origine alimentaire, si la ration est carencée. Cette insuffisance pourrait expliquer qu'au cours d'un premier essai (Geay et al, données non publiées), des taurillons de race tardive n'aient pas réalisé la croissance qu'autorisait le niveau des apports énergétiques et azotés. Les animaux ont reçu en effet un régime d'ensilage de maîs complémenté en amidon et gluten de maïs, caractérisé par une faible teneur en lysine et méthionine (Rulquin et al, 1990).

L'objet de l'expérience présentée a donc été d'étudier l'effet d'un apport de méthionine et de lysine, en supplément de ce type de régime, sur les performances de taurillons.

Matériel et méthodes - Deux lots de 9 taurillons Montbéliards, âgés de 5 mois et pesant $192 \pm 2 \mathrm{~kg}$ en moyenne, ont été mu- nis d'un cathéter au niveau de l'abomasum, relié à une pompe péristaltique. Alimentés à volonté avec un régime constitué de $77 \%$ d'ensilage de maïs et $23 \%$ d'un aliment concentré $176 \%$ de maïs, $18 \%$ de gluten meal, $6 \%$ d'urée) ils ont reçu par infusion soit de la méthionine (MET) et de la lysine - $\mathrm{HCl}$ à raison de $0,21 \%$ de la MSI pour chacune d'elle (lot $M L$ ), soit un même apport d'azote sous forme de glutamate de sodium (lot témoin, TE). L'expérience s'est déroulée au cours d'une période de 2 mois durant laquelle le potentiel de croissance musculaire de ces animaux est connu pour être le plus élevé.

Un certain nombre d'animaux ayant arraché leur cathéter au cours de l'essai, les résultats ne concernent que 9 et 7 animaux respectivement pour les lots (TE) et (ML) durant les 35 premiers j, puis respectivement 5 et 6 animaux durant les 23 j suivants. La croissance journalière a été calculée par régression du poids en fonction du temps à partir de pesées effectuées 2 j consécutifs à la même heure à $0,21,35$ et $58 \mathrm{j}$. Les quantités ingérées ont été mesurées tous les jours. Le bilan azoté de 5 animaux de chaque lot a été mesuré durant $10 \mathrm{j}$, au cours de chacune des périodes. La teneur en urée et en azote $\alpha$-aminé plasmatique de chaque animal a été mesurée en période préexpérimentale, au $30^{\ominus}$ et au $55^{\ominus} \mathrm{j}$ d'expérience. Une analyse de variance a été réalisée sur l'ensemble des résultats à l'aide du logiciel SAS (1987). 
Tableau I. Effets d'un apport de méthionine et de lysine dans la caillette sur les performances de jeunes bovins.

\begin{tabular}{|c|c|c|c|c|}
\hline \multirow{2}{*}{$\begin{array}{l}\text { Durée (j) } \\
\text { Lot } \\
\text { Nombre d'animaux }\end{array}$} & \multicolumn{2}{|c|}{$\begin{array}{c}\text { Période } 1 \\
35\end{array}$} & \multicolumn{2}{|c|}{$\begin{array}{c}\text { Période } 2 \\
23\end{array}$} \\
\hline & $\begin{array}{c}\text { TE } \\
9\end{array}$ & $\mathrm{ML}_{7}$ & $\underset{5}{\mathrm{TE}}$ & $\underset{6}{M L}$ \\
\hline \multirow{3}{*}{ 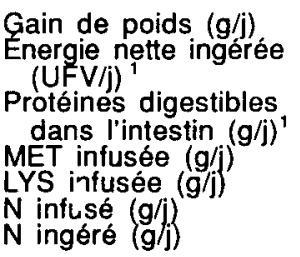 } & $1139 \pm 280$ & $1349 \pm 300$ & $928 \pm 100$ & $1135 \pm 400$ \\
\hline & $2,5 \pm 0,2$ & $2,6 \pm 0,1$ & $2,2 \pm 0,1$ & $2,8 \pm 0,3$ \\
\hline & $\begin{array}{r}328 \pm 25 \\
0,0 \\
0,0 \\
3,3 \pm 0,3 \\
129,5 \pm 9,4\end{array}$ & $\begin{array}{r}328 \pm 34 \\
13,4 \pm 1,4 \\
13,2 \pm 1,4 \\
3,7 \pm 0,4 \\
131,3 \pm 13,0\end{array}$ & $\begin{array}{c}321 \pm 6 \\
0,0 \\
0,0 \\
3,6 \pm 0,1 \\
133,9 \pm 2,5\end{array}$ & $\begin{array}{c}339 \pm 43 \\
14,1 \pm 2 \\
13,9 \pm 1,2 \\
3,9 \pm 0,6 \\
141,9 \pm 17,1\end{array}$ \\
\hline \multirow{2}{*}{$\begin{array}{l}\text { Bilans azotés } \\
N \text { ingéré }(\mathrm{g} / \mathrm{j}) \\
\mathbb{N} \text { urinaire }(\mathrm{g} / \mathrm{j}) \\
\mathbb{N} \text { retenu } \\
\text { (\% } \mathrm{N} \text { ingéré) }\end{array}$} & $\begin{array}{r}142,9 \pm 5,0 \\
45,2 \pm 5,4^{a}\end{array}$ & $\begin{array}{r}144,3 \pm 15,3 \\
36,9 \pm 5,8^{b}\end{array}$ & $\begin{array}{c}128,7 \pm 10,6 \\
37,3 \pm 2,7\end{array}$ & $\begin{array}{r}139,7 \pm 8,9 \\
35,1 \pm 6,1\end{array}$ \\
\hline & $32,8 \pm 4,4$ & $38,1 \pm 5,1$ & $27,8 \pm 3,8^{a}$ & $33,0 \pm 2,4^{b}$ \\
\hline
\end{tabular}

a. b Les moyennes de la même période et de la même ligne diffèrent significativement $(P<0,05) ;{ }^{1}$ quantités ingérées au-dessus de l'entretien.

Résultats et discussion - L'apport d'AAI n'a pas eu d'effet significatif sur l'appétit. Toutefois, bien que les quantités de méthionine et de lysine ingérées par les animaux témoins aient été voisines des recommandations de Owens (1986), l'infusion d'AAl a eu tendance à accroître le gain de poids vif (200 $\mathrm{g} / \mathrm{j}$ de plus en moyenne pour les 2 périodes, soit $+20 \%$ ) et à améliorer de $17 \%$ l'efficacité alimentaire. De même, pour un apport d' $\mathrm{N}$ dans l'abomasum très voisin, les animaux du lot $M L$ ont eu tendance à avoir une plus faible teneur plasmatique en urée $(12,6$ contre $18,0 \mathrm{mg} / 100 \mathrm{ml}, 2^{e}$ période) et en azote $\alpha$-aminé $(2,1$ contre $2,3 \mathrm{mmol} / \mathrm{l})$, traduisant une meilleure utilisation de l'azote ingéré. Mais, compte tenu du faible nombre d'animaux par lot et de la variabilité des réponses, les effets n'ont pas été significatifs. En revanche, l'infusion d'AAl s'est traduite par une diminution $(P<0,05)$ des pertes d'azote urinaire durant la $1^{\text {re }}$ période et par une augmentation $(P<0,05)$ de la proportion d'N retenu ( $\%$ de l' $N$ ingérée).
En conclusion, cet essai montre que les jeunes bovins en croissance peuvent être sensibles, comme les vaches laitières, à la qualité des acides aminés absorbables dans l'intestin. Avec l'apparition et le développement de l'emploi de sources alimentaires issues de l'agro-industrie, il semble donc nécessaire de préciser le niveau et la nature des besoins en AAl de ces jeunes animaux et de prendre en compte la composition en acides aminés limitants des rations.

Remerciements - Nous remercions la Sté Rhône-Poulenc nutrition animale, qui nous a fourni les acides aminés infusés.

Oke BO, Loerch SC, Deetz LE (1986) J Anim Sci 62, 1101-1112

Owens FN (1986) Florida Nutrition Conference Proceedings, 85-100

Rulquin $\mathrm{H}$, Le Hénaff L, Vérité R (1990) Reprod Nutr DeV (suppl 2) $238 S$

SAS (1987) SAS/STAT Guide for personal computer. SAS Institute Inc, Cary, NC, USA

Vérité R, Michalet-Doreau B, Chapoutot P, Peyraud JL, Poncet C (1987) Bull Tech CRZV INRA, Theix, 70 19-34 